\title{
Como idosos hipertensos e diabéticos que moram sozinhos cuidam desses agravos?
}

\author{
Natália Viega de Souza Schmitz*, Ananyr Porto Fajardo**
}

\section{Resumo}

Atualmente, observa-se, com o aumento da expectativa de vida, um crescimento exponencial da proporção de pessoas idosas. A taxa de fecundidade diminuiu e, com os novos arranjos familiares, o número de idosos que moram sozinhos no Brasil mais do que duplicou entre 1992 e 2012, segundo dados mais recentes. Paralelamente, doenças crônicas, como hipertensão arterial sistêmica e diabetes mellitus, atingem essa população em grande escala, constituindo fator de risco fundamental para o desenvolvimento de doenças cardiovasculares. Os idosos requerem proteção e um olhar atento das políticas públicas e dos serviços de saúde, portanto, passa a ser necessário conhecer sua realidade de vida. Esta investigação objetivou conhecer como idosos hipertensos e diabéticos inscritos no Sistema de Acompanhamento e Cadastramento de Pacientes Hipertensos e Diabéticos do Sistema de Informações do Serviço de Saúde Comunitária, que vivem sozinhos no território adscrito da Unidade de Saúde Vila Floresta/Grupo Hospitalar Conceição, em Porto Alegre, RS, cuidam desses agravos, tendo sido identificados vinte possíveis participantes. Foi desenvolvida uma pesquisa qualitativa, exploratória, cujos dados foram coletados mediante entrevistas semiestruturadas com oito indivíduos. Foram constituídas quatro categorias de análise relacionadas ao autocuidado: rede de apoio, principalmente acionando família e alguns serviços na comunidade, atividades da vida diária, desenvolvidas pelos próprios indivíduos e com auxílio de familiares, estilo de vida, destacando-se a dieta e a realização de caminhadas, terapêutica medicamentosa, evidenciando polifarmácia. Em vista dos resultados, considera-se necessário desenvolver novas estratégias políticas e ações educativas para fortalecimento do autocuidado voltado à prevenção e à promoção da saúde, potencializando as habilidades e os desejos dos sujeitos envolvidos.

Palavras-chave: Saúde do idoso. Autocuidado. Diabetes mellitus. Hipertensão.

* Especialista em Saúde da Família e Comunidade pela Residência Integrada em Saúde do Grupo Hospitalar Conceição. Bacharel em Serviço Social pela Universidade de Passo Fundo. Assistente Social do Serviço Social da Indústria - SESI, Gravataí, RS. E-mail: nati.viega@gmail.com

** Doutora em Educação. Mestre em Odontologia. Odontóloga. Coordenadora adjunta do Mestrado Profissional em Avaliação e Produção de Tecnologias para o Sistema Único de Saúde (MP ATSUS/GHC), Centro de Educação Tecnológica e Pesquisa em Saúde - Escola GHC, Grupo Hospitalar Conceição. Docente da Residência Integrada em Saúde do Grupo Hospitalar Conceição (RIS/GHC). E-mail: aportofajardo@gmail.com

$\rightarrow$ http://dx.doi.org/10.5335/rbceh.v13i2.5776

Recebido em: 02/03/2016. Aceito em: 10/08/2016. 


\section{Introdução}

Com os avanços na área da saúde e as melhorias das condições de vida da população, nas últimas décadas a expectativa de vida aumentou de modo considerável, trazendo a perspectiva da longevidade para a existência humana. De acordo com o Instituto Brasileiro de Geografia e Estatística (IBGE), os idosos já representam mais de $13,7 \%$ da população brasileira e constituem o grupo etário que mais cresce, sendo que as projeções apontam que em 2030 representarão 18,6\% dos habitantes no Brasil (IBGE, 2015). Em 2010, o Rio Grande do Sul passou a ocupar a primeira posição em percentual de população idosa entre todos os estados brasileiros (CLOSS; SCHWANKE, 2012), sendo que 17,3\% da população estão acima da faixa etária dos 60 anos (IBGE, 2015).

Vivenciamos dois processos que impactam o campo da saúde. Por um lado, a transição demográfica caracterizada pela mudança do perfil da população, em que a proporção dos idosos aumenta exponencialmente, por outro, assistimos à transição epidemiológica, na qual as principais causas de morte passaram a ser as doenças e os agravos não transmissíveis, incluindo condições relacionadas ao processo de envelhecimento.

As doenças crônicas não transmissíveis (DCNT), definidas como "qualquer condição prolongada que dure mais do que três meses, geralmente progressivas e não curáveis" (REIS et al., 2013, p. 37), são as que mais acometem a saúde da população. Entre elas, estão a hipertensão arterial sistêmica (HAS) e o diabetes mellitus (DM), que atingem a população idosa em grande escala e constituem fatores de risco (FR) para o desenvolvimento de doenças cardiovasculares (DCV), reconhecidas como a maior causa de mortes no Brasil. Além disso, esses agravos resultam no maior custo referente a internações hospitalares no Sistema Único de Saúde (SUS) (SCHMIDT et al., 2011).

Somando-se a esses fatores, observamos alterações no perfil e na configuração das famílias, como diminuição do número de filhos e o aumento dos divórcios e de casos de viuvez, entre outros fatores que incidem na alteração da constituição familiar. Assim, um fenômeno crescente é o número de idosos que moram sozinhos. No Brasil, entre 1992 e 2012, o percentual aumentou em 215\% (OLIVEIRA; NERI; D'ELBOUX, 2016). Porto Alegre é a $15^{\mathrm{a}}$ capital com maior proporção de pessoas que vivem sozinhas no país, sendo que, dentre os $21,4 \%$ da população que reside em domicílios unipessoais na cidade, $15,1 \%$ são idosos (IBGE, 2010).

Os idosos formam um grupo que requer proteção e olhar atento das políticas públicas e, para que isso seja possível, sua realidade precisa ser conhecida. Nessa direção, constituem-se indagações sobre como as pessoas acima de 60 anos que vivem sozinhas, cuidam de si mesmas ou com quem contam para cuidar da saúde. Devido ao fato de residirem sozinhos, é possível que vivenciem determinados problemas, sendo que 
[...] a realidade destes idosos ainda é pouco conhecida no Brasil, portanto, requer investigações mais frequentes, com o intuito de auxiliá-los no enfrentamento das dificuldades do cotidiano (RAMOS; MENEZES; MEIRA, 2010, p. 45).

Outros autores também apontam a necessidade de investigações relacionadas a essa temática, em função da heterogeneidade da velhice, para que possamos oferecer políticas públicas adequadas a esse segmento (RAMOS; MENEZES; MEIRA, 2010; MEIRELES et al., 2007).

A atenção primária à saúde (APS) é um potente coordenador do cuidado, considerando a capacidade de intervenção com ações preventivas, diagnósticas, de acompanhamento e terapêuticas de HAS e DM. Nesse contexto, a Unidade de Saúde Vila Floresta (USVF) do Serviço de Saúde Comunitária (SSC) do Grupo Hospitalar Conceição (GHC), participa do Sistema de Acompanhamento e Cadastramento de Pacientes Hipertensos e Diabéticos (Hiperdia). Seu objetivo é a redução da morbimortalidade por DCNT, intervindo com "ações de promoção e educação em saúde, detecção precoce, tratamento e acompanhamento das pessoas com HAS, DM e demais FR para DCV" (BRASIL, 2009, p. 11).

Considerando o número de idosos que vivem sozinhos, situação que aumenta a probabilidade de vulnerabilidade por não terem apoio presente direto na unidade doméstica (IBGE, 2010), bem como a dupla condição crônica concomitante, especificamente HAS e DM, é premente conhecer as maneiras como esses enfrentam os referidos agravos.
Diante disso, esta pesquisa objetiva conhecer como os idosos hipertensos e diabéticos inscritos no Hiperdia do Sistema de Informações (SIS) do SSC e que vivem sozinhos no território adscrito da USVF/GHC, Porto Alegre, RS, cuidam desses agravos.

\section{Metodologia}

Entre os dias 2 e 31 de julho de 2014, examinando o banco de dados do SIS SSC do GHC, foram selecionados idosos inscritos no Hiperdia vinculados à USVF. Foram identificados 1.369 usuários, desses 940 eram idosos e desses, 216 apresentavam HAS e DM simultaneamente. A seguir, foram identificados os que moravam sozinhos, totalizando 33 indivíduos. Na consulta aos prontuários de família e com as informações dos agentes comunitários de saúde, foi confirmado o total de vinte sujeitos para composição do universo da pesquisa.

Oito pessoas atenderam aos critérios de inclusão e exclusão, pois duas pessoas recusaram-se a participar e dez não foram encontradas após três visitas consecutivas. Neste artigo, os entrevistados são identificados pela letra $\mathrm{E}$, seguida de um número de ordem atribuído pelas autoras.

Almejando uma abordagem qualitativa, exploratória, entrevistas semiestruturadas foram conduzidas na residência de cada idoso, gravadas em áudio e, posteriormente, transcritas. Os dados sociodemográficos tiveram sua frequência descrita, enquanto as informações abertas foram examinadas 
por meio de análise temática (MINAYO, 2014). A leitura repetida e detalhada do material evidenciou a emergência das seguintes categorias analíticas: rede de apoio, atividades da vida diária, estilo de vida e terapêutica medicamentosa.

Esta pesquisa seguiu a Resolução no ${ }^{-466 / 2012}$ e o projeto foi submetido à apreciação no Comitê de Ética em Pesquisa do Hospital Nossa Senhora da Conceição, tendo sido aprovado sob o Parecer no 260/2014.

\section{Resultados e discussão}

O Quadro 1 apresenta a caracterização sociodemográfica detalhada dos sujeitos entrevistados para esta pesquisa.

Quadro 1 - Características dos entrevistados

\begin{tabular}{|c|c|}
\hline Idade & 71 a 94 anos \\
\hline Gênero & $\begin{array}{l}2 \text { homens } \\
6 \text { mulheres }\end{array}$ \\
\hline Estado civil & $\begin{array}{l}2 \text { solteiros } \\
6 \text { viúvos }\end{array}$ \\
\hline $\begin{array}{l}\text { Número de sujeitos } \\
\text { e respectivo número } \\
\text { de filhos }\end{array}$ & $\begin{array}{l}1 \text { sem filho } \\
2 \text { com um filho } \\
2 \text { com dois filhos } \\
2 \text { com três filhos } \\
1 \text { com nove filhos }\end{array}$ \\
\hline Escolaridade & $\begin{array}{l}2 \text { analfabetos } \\
6 \text { com ensino } \\
\text { fundamental incompleto }\end{array}$ \\
\hline
\end{tabular}

Fonte: dados da pesquisa.

O número de mulheres nesse grupo segue a tendência brasileira e mundial em relação à feminização da velhice (CENTRO INTERNACIONAL DE LONGEVIDADE BRASIL, 2015). Da mesma forma, o baixo grau de escolaridade dos entrevistados reflete a tendência nacional (IBGE, 2010).

As quatro categorias de análise emergentes do exame do material abrangem práticas de autocuidado referidas pelos participantes, problematizadas a seguir.

\section{Rede de apoio}

A categoria rede de apoio abrange os recursos identificados pelos idosos como apoio a ser buscado para questões de saúde. É aspecto importante para os cuidados com a saúde e a qualidade de vida, pressupondo laços de troca entre as pessoas, podendo ser formal ou informal. A rede de apoio informal pode envolver amigos, familiares e vizinhos, já a rede formal é constituída por políticas públicas destinadas aos idosos, serviços e órgãos de defesa de direitos, etc. (SOUZA et al., 2008).

Os entrevistados referiram, principalmente, filhos, irmã e netos, mas também mencionaram a Unidade de Saúde e o Centro Dia do Idoso (CDI). O CDI é um centro de convivência de idosos, em conformidade com a Política Nacional de Assistência Social. Esse serviço oferece "atendimento especializado a famílias com pessoas idosas com deficiência e/ou algum grau de dependência, que tiveram suas limitações agravadas por violações de direitos" (PORTO ALEGRE, 2012, p. 3).

Uma das participantes não teve filhos e nunca foi casada; neste caso, a rede de apoio identificada por ela foi a rede formal, citando os equipamentos sociais: 
Saúde é o posto e o CDI, onde eu tô frequentando (E7).

Essa circunstância demonstra a importância de considerar a condição de viver sozinho no planejamento das políticas públicas direcionadas aos idosos, a fim de atender a situações dela decorrentes.

Outra pessoa informou a inexistência do apoio de terceiros e situações que dificultam a prestação de alguns tipos de auxílio, tendo em vista a característica de violência no território:

Aqui ninguém. Só Deus e o telefone celular. Ligo pra chamar um táxi. Ligar pra onde de noite? Se me dá alguma coisa às $22 \mathrm{~h}, 23 \mathrm{~h}$, vai ligar pra quem? Chama táxi e eles não vêm com medo de ser assaltado (E8).

Nesse sentido, a reflexão sobre um conceito ampliado de saúde se faz necessária, já que há envolvimento de outras áreas importantes para que ela possa de fato ser efetivada, como meio ambiente, saneamento básico, habitação, segurança pública, alimentação, etc. (CAMPOS; BARROS; CASTRO, 2004).

Pensando em idosos que vivem em arranjos domiciliares unipessoais, a gerontologia ambiental contribui para ponderarmos sobre a vivência dos que precisam adaptar-se a essa condição. Entre outras perspectivas, considera que o ambiente exerce uma pressão sobre $o$ indivíduo, que irá responder de acordo com suas características físicas, psicológicas e sociais (BATISTONI, 2014).

A importância da participação da família para o sucesso no tratamento da HAS e do DM está evidenciada na literatura (CONTIERO et al., 2009; NAGAI;
CHUBACI; NERI, 2012). Porém, considerando a fragilidade ou inexistência de vínculos familiares, são necessários investimentos intelectuais e financeiros, a fim de repensar e construir outros modelos que abordem o envelhecimento de forma digna, não depositando apenas na instituição familiar a responsabilidade pelo cuidado com os idosos (DEBERT; SIMÕES, 2011).

Em alguns casos, foram feitas referências a pessoas da família como recurso acionado em caso de necessidades de saúde, incluindo parentes em primeiro e segundo grau:

A minha irmã, ela mora próximo. Eu recorro ali e ela recorre eu (E2).

Ah, mas não tem dúvida né, só aperto o botão e meu filho tá aqui (E3).

O fato de morar sozinho não necessariamente está ligado ao abandono da família ou à ausência de apoio, já que as facilidades de mobilidade e comunicação permitem outros arranjos, mantendo a facilidade de acesso mesmo com o distanciamento físico (DEBERT; SIMÕES, 2011). O contrato informal entre as gerações, segundo o qual os pais cuidam dos filhos e esses cuidam dos pais, é algo que pode ou não ser construído ao longo do tempo. Em pesquisa de Alvarenga et al. (2011), a família foi identificada como a maior fonte de cuidados.

As relações familiares são complexas e dinâmicas, portanto, não podemos fazer generalizações quanto à ideia de proteção e cuidado na família. Essa realidade precisa ser compreendida pelos profissionais de saúde para um melhor acompanhamento ao idoso. 


\section{Atividades da vida diária}

De acordo com Moraes (2012), as atividades de vida diária (AVDs) são fundamentais para a autopreservação e os cuidados de saúde do indivíduo. Caracterizam-se como tarefas do cotidiano classificadas de acordo com o grau de complexidade, a saber: básicas, referindo-se ao autocuidado e à higiene pessoal, como tomar banho, instrumentais, mais complexas e indicadoras da capacidade do idoso para viver sozinho, como preparar alimentos, fazer compras, controlar o dinheiro, usar medicamentos corretamente, realizar trabalhos domésticos, etc., e tarefas avançadas, que são atividades de integração social, como trabalhar, fazer serviço voluntário, dirigir, etc. $\mathrm{O}$ desempenho de atividades específicas no dia a dia com facilidade pode ser determinante para estimular o idoso a sair de casa, mantendo-se socialmente ativo (MORSCH et al., 2015).

Os participantes apontaram que familiares os acompanham em consultas médicas e na realização de exames, também auxiliando nas atividades domésticas ligadas à higiene e à organização do ambiente em que vivem.

Mas ela [filha] é que cuida, limpa pra mim tudo, ajuda (E1).

Se eu vou varrer o pátio, ela vem me ajudar (E2).

Em pesquisa realizada em outros países, nos quais também aumenta $o$ número de idosos que moram sozinhos, muitos deles declararam receber da família diversos tipos de ajuda, inclusive para mobilidade e realização de tarefas domésticas (GRUNDY, 2001).

Identificamos que a dedicação ao espaço que habitam, para além da higiene necessária à manutenção da saúde e à prevenção de algumas doenças, está vinculada à produção de saúde para esses idosos, considerando que essas atividades podem gerar bem-estar e proporcionar olhares positivos diante das situações da vida.

Percebemos que o desempenho dessas atividades é considerado um elemento de cuidado em saúde. Nesse sentido, os sujeitos referiram as AVDs básicas e as instrumentais:

Faço meu serviço todo. Eu lavo, eu passo, eu cozinho. Eu gosto de fazer minhas compras. A doutora perguntou: quem é que faz as tuas compras? Mas eu mesma! Quem é que vai pagar? Eu mesma! Eu mesma vou receber, eu mesma vou pagar (E2).

E no mais eu faço meu serviço de banco, de loja, de farmácia e de médico, tudo, tudo (E3).

Os relatos mostram que realizar essas atividades também é uma forma de manter a capacidade de desempenhar essas funções por conta própria, o que lhes confere maior grau de autonomia, possibilitando concepções sobre si que potencializam suas habilidades, inclusive em relação a morar só:

Eu cuido a alimentação, eu caminho, mais a atividade toda que eu tenho de uma dona de casa (E5).

Uma vez por semana eu carrego a máquina, estendo lá nos fundos, isso também faz parte do exercício, botar a roupa, erguer (E3). 
Percebe-se nos relatos a satisfação dos idosos consigo mesmo por perceber a habilidade de realizar as AVDs como uma forma de se manter independentes. Nesse sentido, concordamos com Teixeira (2002) sobre a possibilidade de tomar conta de si mesmo ser aumentada pelo bem-estar. Assim, observamos uma relação cíclica entre capacidade funcional, empoderamento e autocuidado.

\section{Estilo de vida}

Um dos principais determinantes da morbidade e da prevalência das DCNT é o estilo de vida (BRASIL, 2014). Visto que os hábitos alimentares e a realização de atividades físicas estão intimamente relacionados aos cuidados de HAS e $\mathrm{DM}$, a alimentação é fundamental para manutenção, produção e recuperação da saúde, influenciando de forma significativa no envelhecimento saudável (CAMPOS et al., 2006).

Em relação aos portadores de HAS e DM, existem recomendações específicas sobre a dieta, como a diminuição do consumo de gorduras de origem animal, açúcar e sal e o incentivo à ingestão de legumes, verduras, etc. (MARTINS et al., 2010). Referente à alimentação e à prática de atividades físicas, os sujeitos apontaram os cuidados nutricionais como aspecto de grande relevância para HAS e DM, além da atividade física, sendo a caminhada a forma de exercício mais comentada.

Os cuidados com a alimentação foram referidos como aspecto de autocuidado:
Eu me cuido muito com comida, porque a gente sabe que não pode abusar com nada quando a gente tem problema, pode ter piora da diabete, é coisa séria (E4).

Observamos que esses indivíduos estão informados acerca das orientações alimentares direcionadas para diabéticos e hipertensos, pois relatam praticar cuidados com a sua alimentação em função de sua condição patológica.

Não como fritura, faz anos que eu não como. Não como porque eu tenho pressão, colesterol, diabetes (E6).

Sal eu não posso por causa da hipertensão, se tem ácido eu não posso por causa da gastrite, então eu tenho que comer uma massa ou arroz; se eu como massa eu não como arroz por causa da diabete (E8).

Predominaram os relatos quanto aos cuidados com a redução da ingestão de açúcares, sal e gordura, bem como com a manutenção do consumo de frutas, legumes e verduras.

Agora a carne de porco só de tempos em tempos, porque é uma carne muito gordurosa. [...] E é isso, feijão, arroz, bastante salada verde, salada crua (E3).

Como às vezes de 3 a 4 frutas diversas por dia e bebo muita água (E5).

Não posso comer salame, não posso comer patê, tudo tem sal (E8).

Com relação ao preparo dos alimentos, um dos idosos tem a refeição do almoço encomendada por serviço de vianda e outro se alimenta no CDI. Porém, na maioria dos casos, os próprios idosos preparam sua alimentação. Um entrevistado explicou que passou a prepará-la com vista a refeições mais saudáveis: 
Eu faço a minha comida, boto sal, o limite mínimo por causa da pressão. Eu comia aqui no buffet da frente, mas o feijão salgado, a massa salgada, a carne salgada, tudo salgado, aí não podia comer (E8).

Outra pessoa informou que não tem restrições alimentares por não sentir nenhum sintoma imediato após a ingestão dos alimentos:

Como qualquer comida que é de mastigar. Tô sentada lá, levanto, pego um pedaço de carne, pego uma morcilha [...]. E graças a Deus nunca me fez mal [...]. A comida eu não cuido porque graças a Deus, eu como e nunca me fez mal, nada. Agora se me fizer mal já, daquilo ali eu já recuo também (E2).

Essa situação indica a importância de os profissionais de saúde problematizarem os diferentes aspectos dos agravos com o usuário, de modo a ampliar suas reflexões sobre suas condições de saúde. Compreendemos que as intervenções com enfoque na promoção do autocuidado devem considerar o saber-experiência próprio, de acordo com o conceito freireano (FREIRE, 1996), construindo novas possibilidades e modos de viver.

A cultura popular apropriada do conhecimento científico (e vice-versa) em relação ao tratamento da HAS também foi evidenciada:

Chuchu é bom pra normalizar a pressão. E a água do chuchu eu guardo pra tomar quando a pressão quer subir demais, eu tomo um gole, dois e normaliza (E8).

Dessa forma, "[...] conhecer hábitos, culturas e crenças dos indivíduos com hipertensão" (LOPES et al., 2008, p. 205) é útil para o manejo da doença, pois o processo de autocuidado inclui também o conhecimento originado nas comunidades.

Embora menos frequente, a atividade física foi citada pelos participantes como uma das práticas de cuidado no tratamento de HAS e DM. A atividade mais apontada foi a caminhada. Seguem algumas falas quanto à atividade física:

Eu gosto de caminhar (E1).

Eu caminho, né [...]. Eu tenho uma bicicleta ergométrica que é meu exercício (E3).

Marquei já exercícios, alongamento com o [profissional de educação física] aqui no Cecoflor [Centro Comunitário Floresta] (E7).

A prática de atividades físicas regulares é um dos hábitos recomendados nos tratamentos de HAS e DM (BRASIL, 2014). As recomendações da Organização Mundial da Saúde para idosos incluem 150 minutos de atividade física aeróbia moderada semanal, além de fortalecimento muscular duas vezes por semana (WORLD HEALTH ORGANIZATION, 2010).

Convém apontar que investimentos em políticas públicas que assegurem à população o direito ao lazer e à prática de atividade física de qualidade e com segurança desonerariam o SUS (BIELEMANN; KNUTH; HALLAL, 2010), em função do potencial da influência dessas atividades sobre os mecanismos patológicos da HAS e do DM.

O tratamento não medicamentoso ainda é adotado de forma incipiente, pois a mudança de hábitos envolve uma série de dificuldades, entre elas, o abandono de comportamentos que outrora geravam 
prazer (LOPES et al., 2008). Assim, aflora a necessidade de adaptações que exijam dos idosos uma abertura para a possibilidade de novos modos de ser e viver.

Tendo em vista as diversas instruções quanto à realização de atividade física para uma vida saudável, problematizamos alguns aspectos que dificultam seu cumprimento. $\mathrm{O}$ incentivo à prática regular dessas atividades ainda ocorre de forma genérica, desconsiderando, muitas vezes, o contexto e os recursos disponíveis aos idosos. Quanto aos espaços públicos e aos equipamentos sociais destinados à prática de atividade física, ainda são insuficientes e, muitas vezes, apresentam condições inadequadas e inseguras à população.

As intervenções com o objetivo de motivar mudanças no estilo de vida são indispensáveis no controle e tratamento das doenças crônicas (OLINDA; SILVA, 2009). No entanto, há que se ponderar as condições, o contexto, as dificuldades e as potencialidades do idoso nesse processo.

\section{Terapêutica medicamentosa}

A terapêutica medicamentosa ocorre pela administração de fármacos para $o$ tratamento de doenças e seus sintomas. No contexto da população idosa acometida por DCNT, essa questão apresenta-se como uma preocupação pelos cuidados que devem ter no tratamento, além do uso dos diversos medicamentos (VIEIRA; CASSIANI, 2014). No caso dos sujeitos desta pesquisa, a atividade de autocuidado foi a mais enfatizada nos relatos. $\mathrm{O}$ número das diversas medicações utiliza- das variou de três a nove, e os entrevistados indicaram seu uso como fundamental no tratamento de HAS e DM:

Eu tomo o meu medicamento tudo corretamente, nos horários certos (E5).

Eu brinco que vocês andam com a gasolina e eu ando com isso aqui [mostra caixas de medicamento] (E6).

A condição de polifarmácia dos participantes também chamou a atenção:

Tomo cinco. Tomo pra ficar boa (E1).

É uma série de remédios controlados que eu tomo três vezes ao dia (E5).

Eu tomo, no dia todo, dez comprimidos, e não posso ficar sem tomar (E8).

Podemos dizer que o tratamento medicamentoso exige, além de organização disciplinada com os horários, o controle dos diversos tipos de medicamentos. Cabe apontar a necessidade de um olhar atento aos riscos da polifarmácia, haja vista a possibilidade de interações medicamentosas, reações adversas e erros ao administrar a quantidade de fármacos (VIEIRA; CASSIANI, 2014).

Cabe problematizarmos a influência "do poder da indústria farmacêutica e do marketing dos medicamentos e a medicalização presente na formação de parte expressiva dos profissionais da saúde" (SECOLI, 2010, p. 137). Podemos dizer que esses trabalhadores podem ter suas práticas regidas, em grande parte, pela lógica da medicalização, que, por seu turno, continua sendo a forma de cuidado predominante a ser oferecido para os usuários (COUTINHO et al., 2013). 
Outro aspecto que reforça essa lógica é a diversidade de propagandas e reportagens na mídia que difundem a ideia dos medicamentos como componente essencial no processo do cuidado (FERRAZ; LERNER, 2013). Essa situação pode denotar a relevância atribuída à terapêutica farmacológica pelos serviços de saúde e sua influência sobre a cultura dos pacientes, possivelmente, sobrepondo-se a outros cuidados de diferentes ordens para enfrentamento de patologias.

\section{Considerações finais}

Este processo investigativo permitiu perceber que os entrevistados cuidam da HAS e do DM de diversas maneiras e contam com rede de apoio formal e/ ou informal. A realização das AVDs foi considerada elemento importante como cuidado, além do estilo de vida, que inclui hábitos alimentares, como redução de açúcar e sal e aumento do consumo de frutas e verduras, e prática de atividades físicas, sendo a caminhada a mais observada. A terapêutica medicamentosa foi a mais enfatizada pelos participantes.

Diante desses resultados, torna-se interessante pensar em alguns elementos no contexto do envelhecimento populacional. Quanto à rede de apoio, alguns equipamentos sociais e também a família foram citados como fonte de ajuda no processo de cuidado. Considerando que os futuros idosos terão menos filhos e, portanto, menos chances de contar com o apoio da família, é necessário refletir acerca de novas estratégias, na perspectiva de incentivo ao protagonismo dos ido- sos, por meio do qual eles sejam encorajados a desenvolver suas potencialidades. Também fica evidente a relevância de equipamentos sociais que proporcionem um ambiente apropriado para atender às necessidades desse grupo populacional, tendo em vista sua autonomia.

Além disso, as políticas sociais devem ser elaboradas e colocadas em prática com vistas à educação para uma vida saudável e ativa, favorecendo a funcionalidade dos idosos pelo maior tempo possível. Apesar da importância do estilo de vida no tratamento de HAS e DM, o incentivo efetivo do poder público e dos profissionais de saúde para a adesão a hábitos saudáveis ainda é insuficiente, além da carência de espaços seguros para a prática de atividades físicas.

A farmacoterapia continua tendo maior destaque dentre as medidas promotoras de saúde e qualidade de vida para a população idosa. É necessário desenvolver ações educativas para um autocuidado voltado à prevenção e à promoção da saúde, potencializando as habilidades e os desejos dos sujeitos, de maneira a racionalizar a dependência de recursos farmacológicos.

Uma possível limitação deste estudo foi o número de respondentes. Contudo, considera-se que a interação com apenas oito indivíduos em vez de vinte, como indicavam os prontuários de família, demonstra a dinâmica do trabalho em APS, pois o andar da vida nem sempre consegue ser acompanhado e registrado pelos trabalhadores em atividade. Sendo assim, acredita-se ser importante ampliar este estudo, possivelmente, em 
territórios diversos, buscando a identificação de outros aspectos que indiquem a qualidade de vida de idosos que vivem sozinhos, almejando qualificar seu viver e aprimorar a atenção prestada pelos serviços de saúde aos quais estão vinculados.

\section{Agradecimentos}

Agradecemos aos participantes por terem disponibilizado seu tempo e suas vivências para viabilizar esta produção, à Patrícia de Lima pela parceria na coleta de dados e ao Ministério da Saúde pela bolsa-residência, que permitiu dedicação exclusiva da primeira autora à Residência Integrada em Saúde do Grupo Hospitalar Conceição.

\section{How hypertensive and diabetic elderly people who live alone take care of these health conditions}

\section{Abstract}

With the present increase of life expectancy, there is an exponential increment in the proportion of elderly people. The fecundity rate decreased and, due to new family organization, the number of aged people who live alone has more than doubled between 1992 and 2012 in Brazil. Simultaneously, chronic conditions like Systemic Hypertension and Diabetes Mellitus are widespread in the older population, being major risk factors for the development of cardiovascular diseases. Senior citizens deserve protection and a close look from public policies and health services; thus, it is necessary to grasp their reality of life. This investigation aimed to know how hypertensive and diabetic elderly people, registered in the Follow-up and Registering System of Hypertensive and Diabetic Patients System (Hiperdia) of the Information System of Vila Floresta/Grupo Hospitalar Conceição, Porto Alegre/RS, living alone within the territory under its responsibility take care of these conditions. Twenty people were identified as possible participants and the qualitative, exploratory research was conducted with eight seniors, who participated of semi-structured interviews. Four analytical categories concerning self care were identified: support network, mainly identified as family and community resources; activities of daily living, developed by the individuals and with help from family members; life style, identified as diet and walking; and drug therapy, evidencing polypharmacy. In face of these results, a need of new political strategies and educational actions was identified, in order to strengthen the self care aimed to prevention and health promotion, improving the potential of the skills and desires of the involved subjects.

Keywords: Diabetes mellitus. Health of the elderly. Hypertension. Self care.

\section{Referências}

ALVARENGA, Márcia R. M. et al. Rede de suporte social do idoso atendido por equipes de Saúde da Família. Ciência \& Saúde Coleti$v a$, Rio de Janeiro, v. 16, n. 5, p. 2.603-2.611, 2011. Disponível em: <http://www.scielo.br/ scielo.php?script=sci_arttext\&pid=S1413$-81232011000500030 \& \operatorname{lng}=\mathrm{en} \& \mathrm{nrm}=\mathrm{iso} \& \mathrm{tl}$ $\mathrm{ng}=\mathrm{pt}>$. Acesso em: 10 nov. 2015.

BATISTONI, Samila S. T. Gerontologia ambiental: panorama de suas contribuições para a atuação do gerontólogo. Revista Brasileira de Geriatria e Gerontologia, Rio de Janeiro, v. 17, n. 3, p. 647-657, 2014. Disponível em: <http:// www.scielo.br/pdf/rbgg/v17n3/1809-9823rbgg-17-03-00647.pdf>. Acesso em: 5 set. 2015. 
BIELEMANN, Renata M.; KNUTH, Alan G.; HALLAL, Pedro C. Atividade física e redução de custos por doenças crônicas ao Sistema Único de Saúde. Revista Brasileira de Atividade Física \& Saúde, Pelotas, v. 15, n. 1, p. 9-14, 2010. Disponível em: <http://periodicos.ufpel.edu.br/ojs2/index.php/RBAFS/ article/view/674/689>. Acesso em: 15 ago. 2015.

BRASIL. Ministério da Saúde. Grupo Hospitalar Conceição. Protocolo de Hipertensão Arterial Sistêmica para a Atenção Primária em Saúde. Porto Alegre: Hospital Nossa Senhora da Conceição, 2009. (Organização de Sandra Rejane Soares Ferreira et al.; Ilustrações de Maria Lúcia Lenz).

. Ministério da Saúde. Secretaria de Atenção à Saúde. Departamento de Atenção Básica. Estratégias para o cuidado da pessoa com doença crônica. Brasília: Ministério da Saúde, 2014. Disponível em: <http://bvsms. saude.gov.br/bvs/publicacoes/estrategias_cuidado_pessoa_doenca_cronica_cab35.pdf $>$. Acesso em: 14 set. 2015.

CAMPOS, Gastão W.; BARROS, Regina. B. de; CASTRO, Adriana M. de. Avaliação de política nacional de saúde. Ciência \& Saúde Coletiva, Rio de Janeiro, v. 9, n. 3, p. 745-749, 2004. Disponível em: <http://www.scielo.br/ pdf/csc/v9n3/a20v09n3.pdf>. Acesso em: 2 out. 2015.

CAMPOS, Marta A. G. et al. Estado nutricional e fatores associados em idosos. Revista da Associação Médica Brasileira, São Paulo, v. 52, n. 4, p. 214-221, 2006. Disponível em: $<$ http://www.scielo.br/scielo.php?pid=s0104$-42302006000400019 \&$ script $=$ sci_arttext $>$. Acesso em: 10 out. 2015.

CLOSS, Vera E.; SCHWANKE Carla H. A. A evolução do índice de envelhecimento no Brasil, nas suas regiões e unidades federativas no período de 1970 a 2010. Revista Brasileira de Geriatria e Gerontologia, Rio de Janeiro, v. 15, n. 3, p. 443-458, 2012.
CONTIERO, Ana P. et al. Idoso com hipertensão arterial: dificuldades de acompanhamento na Estratégia Saúde da Família. Revista Gaúcha de Enfermagem, Porto Alegre, v. 30, n. 1, p. 62-70, 2009. Disponível em: <http://seer.ufrgs.br/RevistaGauchadeEnfermagem/article/view/4227>. Acesso em: 10 ago. 2015.

COUTINHO, Aline T. et al. Integralidade do cuidado com o idoso na Estratégia de Saúde da Família: visão da equipe. Escola Anna Nery, Rio de Janeiro, v. 17, n. 4, p. 628-637, set./dez. 2013. Disponível em: <http://www. scielo.br/pdf/ean/v17n4/en_1414-8145ean-17-04-0628.pdf>. Acesso em: 16 out. 2015.

DEBERT, Guita G.; SIMÕES, Júlio A. Envelhecimento e velhice na família contemporânea. In: FREITAS, Elizabete V. de et al. Tratado de geriatria e gerontologia. 3. ed. Rio de Janeiro: Guanabara Koogan, 2011. p. 1571-1579.

FERRAZ, Luiz M. R.; LERNER, Katia. Sob o signo do patológico na mídia: risco, cuidado crônico e medicalização nos discursos de Veja e Época. In: CONGRESSO BRASILEIRO DE CIÊNCIAS DA COMUNICAÇÃO, 36, 4 a 7 set. 2013. Manaus, 2013. Disponível em: <http://www.intercom.org.br/papers/nacionais/2013/resumos/R8-1855-1.pdf>. Acesso em: 25 out. 2015 .

FREIRE, Paulo. Pedagogia da autonomia: saberes necessários à prática educativa. São Paulo: Paz e Terra, 1996.

GRUNDY, Emily. Living arrangements of older persons and family support in more developed countries. Population Bulletin of the United Nations, n. 42-43, 2001. Special issue Disponível em: <http://www.un.org/ esa/population/publications/bulletin42_43/ bulletin42_43.htm>. Acesso em: 12 set. 2015.

INSTITUTO BRASILEIRO DE GEOGRAFIA E ESTATÍSTICA. Censo Demográfico 2010. Famílias e domicílios: resultados da amostra. Rio de Janeiro: IBGE, 2010. 
. Síntese de indicadores sociais: uma análise das condições de vida da população brasileira. Rio de Janeiro: IBGE, 2015. Disponível em: <http://biblioteca.ibge.gov. br/visualizacao/livros/liv95011.pdf $>$. Acesso em: 17 jul. 2016.

CENTRO INTERNACIONAL DE LONGEVIDADE BRASIL. Envelhecimento ativo: um marco político em resposta à revolução da longevidade. Rio de Janeiro, 2015.

LOPES, Mislaine C. de L. et al. O autocuidado em indivíduos com hipertensão arterial: um estudo bibliográfico. Revista Eletrônica de Enfermagem, Goiânia, v. 10, n. 1, p. 198211, 2008. Disponível em: <https://www.fen. ufg.br/fen_revista/v10/n1/v10n1a18.htm>. Acesso em: 20 nov. 2015.

MARTINS, Maria do P. S. C. et al. Consumo alimentar, pressão arterial e controle metabólico em idosos diabéticos hipertensos. Revista Brasileira de Cardiologia, Rio de Janeiro, v. 23, n. 3, p. 162-170, maio/jun. 2010. Disponível em: <http://sociedades.cardiol.br/ socerj/revista/2010_03/a2010_v23_n03_completa.pdf\#page $=14>$. Acesso em: 2 set. 2015.

MEIRELES, Viviani C. et al. Características dos idosos em área de abrangência do programa Saúde da Família na Região Noroeste do Paraná: contribuições para a gestão do cuidado em enfermagem. Saúde e Sociedade, São Paulo, v. 16, n. 1, p. 69-80, jan./abr. 2007. Disponível em: <http://www. scielo.br/pdf/sausoc/v16n1/07/pdf>. Acesso em: 3 set. 2015 .

MINAYO, Maria C. de S. O desafio do conhecimento: pesquisa qualitativa em saúde. 14. ed. São Paulo: Hucitec, 2014.

MORAES, Edgar N. Atenção à saúde do idoso: aspectos conceituais. Brasília: Organização Pan-Americana da Saúde, 2012.

MORSCH, Patricia et al. Características clínicas e sociais determinantes para o idoso sair de casa. Cadernos de Saúde Pública, Rio de Janeiro, v. 31, n. 5, p. 1.025-1.034, maio 2015.
NAGAI, Paula A.; CHUBACI, Rosa Y. S.; NERI, Anita L. Idosos diabéticos: as motivações para o autocuidado. Revista Temática Kairós Gerontologia, São Paulo, v. 15, n. 6, p. 407-434, dez. 2012. Disponível em: <http:// revistas.pucsp.br/index.php/kairos/article/ view/17315>. Acesso em: 20 nov. 2015.

OLINDA, Querubina B.; SILVA, Carlos, A. B. da. As doenças crônicas matam no silêncio. $R e$ vista Brasileira em Promoção da Saúde, Fortaleza, v. 22, n. 3, p. 135-136, 2009. Disponível em: <http://www.redalyc.org/pdf/408/40812007001. pdf>. Acesso em: 1 out. 2014.

OLIVEIRA, Déborah Cristina de; NERI, Anita Liberalesso; D'ELBOUX, Maria José. Revista Brasileira de Enfermagem, Brasília, v. 69 , n. 3, p. 566-573, maio/jun. 2016. Disponível em: <http://dx.doi.org/10.1590/0034-7167.2016690321i>. Acesso em: 28 jul. 2016.

PORTO ALEGRE. Fundação de assistência social e Cidadania. Proteção social especial de média complexidade. Projeto Centro Dia do Idoso. 2012. Disponível em: <http://lproweb. procempa.com.br/pmpa/prefpoa/fasc/usu_doc/ projeto_tecnico_centro_dia_do_idoso_sul_centro_sul.pdf>. Acesso em: 18 nov. 2015.

RAMOS, José L. C.; MENEZES, Maria do R. de; MEIRA, Edméia C. Idosos que moram sozinhos: desafios e potencialidades do cotidiano. Revista Baiana de Enfermagem, Salvador, v. 24, n. 1, 2, 3, p. 43-54, jan./dez. 2010. Disponível em: <http://www.portalseer.ufba.br/index.php/enfermagem/article/ view/5527/3979>. Acesso em: 7 set. 2014.

REIS, Luciana A. dos et al. O envelhecimento populacional brasileiro e as doenças crônico-degenerativas e não transmissíveis, 2013. In: IMPERATORI, Adriano et al. (Org.). Doenças crônicas: repercussões e intervenções. Passo Fundo: Berthier, 2013. p. 35-48.

SCHMIDT, Maria I. et al. Doenças crônicas não transmissíveis no Brasil: carga e desafios atuais. 2011. (Séries Saúde no Brasil, v. 4). Disponível em: <http://www.uniad.org.br/ desenvolvimento/images/stories/pdf/brazilpor41.pdf>. Acesso em: 31 out. 2014. 
SECOLI, Silvia R. Polifarmácia: interações e reações adversas no uso de medicamentos por idosos. Revista Brasileira de Enfermagem, Brasília, v. 63, n. 1, p. 136-140, jan./fev. 2010. Disponível em: <http://www.scielo.br/ pdf/reben/v63n1/v63n1a23.pdf $>$. Acesso em: $1^{\circ}$ nov. 2015.

SOUZA, Edinilsa R. de et al. Rede de proteção aos idosos do Rio de Janeiro: um direito a ser conquistado. Ciência \& Saúde Coletiva, Rio de Janeiro, v. 13, n. 4, p. 1.153-1.163, 2008. Disponível em: <http://www.scielosp. org/pdf/csc/v13n4/11.pdf>. Acesso em: 7 jul. 2015.

TEIXEIRA, Mirna B. Empoderamento de idosos em grupos de promoção da saúde. Dissertação (Mestrado em Saúde Pública) - Escola Nacional de Saúde Pública Sergio Arouca, Fundação Oswaldo Cruz, Rio de Janeiro, 2002. Disponível em: <http://portalteses.icict. fiocruz.br/pdf/FIOCRUZ/2002/teixeirambm/ capa.pdf>. Acesso em: 17 jul. 2016.

VIEIRA, Liliana B.; CASSIANI, Sílvia H. de B. Avaliação da adesão medicamentosa de pacientes idosos hipertensos em uso de polifarmácia. Revista Brasileira de Cardiologia, Rio de Janeiro, v. 27, n. 3, p. 195-202, maio/jun. 2014. Disponível em: <http:// www.rbconline.org.br/wp-content/uploads/ Art_181_Liliana_Vieira_Artigo_Original. pdf>. Acesso em: 18 nov. 2015.

WORLD HEALTH ORGANIZATION. $E n$ velhecimento ativo: uma política de saúde. Brasília: Organização Pan-Americana de Saúde, 2010. 